\title{
Abdominal aortic aneurysm repair and the horseshoe kidney
}

\author{
DANIEL J. WATERS, D.O. \\ Cleveland, Ohio \\ ALAN N. ESPER, D.O \\ Erie, Pennsylvania \\ WILLIAM E. STANLEY, D.O., FACOS \\ Des Moines, Iowa
}

The coexistence of

abdominal aortic aneurysm and horseshoe kidney is uncommon. In the case reported here, this condition was encountered when the patient was undergoing aortic reconstruction for aneurysm. Surgical repair in such circumstances remains a technical challenge because of the vagaries of arterial supply, coupled with the spatial constraints on aortic exposure and control. Preoperative urography or arteriography may facilitate operative planning. Whether the situation is encountered knowingly or unexpectedly, extreme care must be taken to preserve segmental renal arterial supply both during and after aortic reconstruction.

A horseshoe kidney is an anatomic anomaly in which the lower renal poles are fused and embryologic ascension is incomplete. Its coexistence with abdominal aortic aneurysm is an uncommon clinical problem. A literature review yielded 80 previously reported cases. ${ }^{1-22}$ The association is thought to be one of chance. Because of the often variable renal blood supply and the location of the renal symphysis, aortic reconstruction in these patients presents a significant technical challenge, even when the anomaly is diagnosed preoperatively. When it is encountered at surgery, care must be exercised to preserve renal blood flow, while, at the same time, adequate exposure for aortic control and repair must be obtained.

We present a case of horseshoe kidney which was discovered at laparotomy.

\section{Report of case}

A 62-year-old white man presented with back pain. Abdominal examination revealed a pulsatile mass. Ultrasonography demonstrated an infrarenal abdominal aortic aneurysm, which measured $4 \mathrm{~cm}$. in greatest diam- eter; no other abnormalities were noted. Aneurysm repair was recommended, and the patient underwent extensive preoperative evaluation, including coronary angiography, which showed significant two-vessel arterial stenosis.

The past surgical history was remarkable for subtotal gastrectomy for ulcer disease 8 years prior to admission. No anatomic abnormalities had been noted at that time. There was no history of recent urinary tract infection, pyelonephritis, or renal calculi.

The results of complete blood cell count, serum electrolyte determination, and urinalysis were normal. The serum creatinine value was $0.6 \mathrm{mg}$./dl. The chest $\mathrm{x}$-ray film yielded normal results.

The patient underwent protective coronary artery bypass grafting, from which he recovered uneventfully. He returned approximately 3 months later for elective aneurysm repair. Preoperative laboratory testing yielded normal results.

At laparotomy, when the retroperitoneum was incised, a dense, parenchymatous structure was noted overlying the upper portion of the aortic aneurysm at the level of the third lumbar vertebra. After careful dissection, this was identified as the symphysis of a horseshoe kidney. There appeared to be at least one anomalous artery coursing to the kidney isthmus, with its origin at or near the apparently normal right and left renal arteries (Fig. 1). This accessory vessel originated above the neck of the aneurysm. The renal isthmus was mobilized and encircled with a Penrose drain. No other anomalous vessels could be identified. The ureters were not encountered in the dissection.

Proximal aortic control was obtained cephalad to the isthmus, but below the normal and accessory renal arteries. The isthmus was then displaced superiorly, and, after control of the distal aorta was obtained, the aneurysm was opened and the clot debris was removed. Because of the aneurysmal dilatation of the common iliac arteries, the decision was made to proceed with aortobifemoral reconstruction with a woven Dacron graft. Distal aortic exclusion above the iliac bifurcation was obtained by suture ligation. The renal symphysis was retracted superiorly and the proximal anastomosis was constructed. The limbs of the $v$-graft were anastomosed to the common femoral arteries in the standard fashion. The reconstructed abdominal aorta is shown in Figure 2. The wall of the aneurysm was closed over the proximal anastomosis, and the retroperitoneum and abdominal 

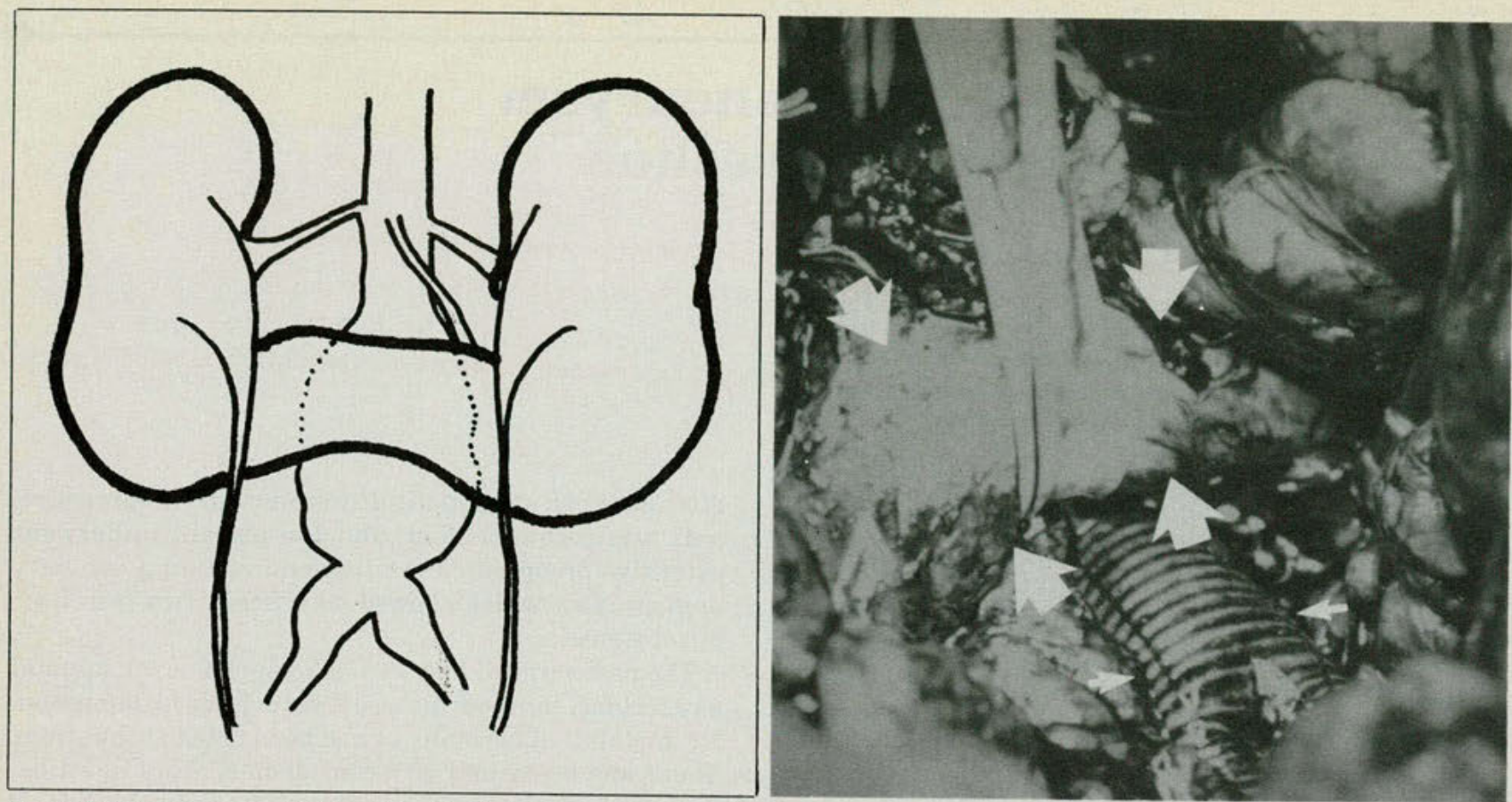

Fig. 1: Schematic representation of anatomic features encountered in the reported case. A single anomalous renal vessel coursing laterally to the renal isthmus was identified. Fig. 2: The reconstructed abdominal aorta. The isthmus of the horseshoe kidney (large arrow) is encircled with a Penrose drain. The bifurcated graft (small arrows) passes posterior to the isthmus.

wall were closed in a standard manner.

The patient had an uneventful recovery and was discharged on the tenth postoperative day. Urinary output and serum creatinine values during the postoperative period remained normal.

\section{Discussion}

Abdominal aortic aneurysm in the presence of horseshoe kidney is an unusual surgical problem. Whether encountered before or during laparotomy for aneurysm repair, it presents a complex technical problem for the vascular surgeon.

Our review of the literature revealed 80 previous cases, ${ }^{1-22}$ with this patient representing the eightyfirst. This number is more than double that found by Bietz and Merendino, ${ }^{1}$ whose 1975 review yielded 32 cases, to which they added an additional 2 cases.

Horseshoe kidney is not a rare anomaly. Its incidence has been estimated at 1 in 400 persons. ${ }^{23}$ The definitive kidneys arise from the primitive metanephros in the fetal pelvis. From here they ascend to their normal lumbar position. While ascending, they pass through the "fork" formed by umbilical arteries. It is thought that the horseshoe configuration is caused by the kidneys being pushed close together during their ascent so that the lower poles fuse. The level of the symphysis, or fused isthmus, usually is at the lower lumbar vertebrae, because the origin of the inferior mesenteric artery prevents further cephalad migration. ${ }^{24}$ Normal rota- tion of the renal axis is inhibited by lower pole fusion so that the renal pelvis remains oriented anteriorly. ${ }^{25}$ The ureters usually course anteriorly to the isthmus. ${ }^{24,25}$

The variations in arterial supply to the horseshoe kidney were categorized by Graves ${ }^{26}$ into six basic patterns. This work was supplemented later by Boatman and associates ${ }^{25}$ at the University of Iowa. The vascular supply may consist of normally paired renal arteries or one or more accessory vessels arising from the infrarenal aorta and/or the common iliac, internal iliac, and middle sacral arteries. Graves's resin cast studies demonstrated that each artery supplied a distinct renal segment, with no collateral circulation. ${ }^{25,26}$ When aneurysmal dilatation of the aorta occurs in this setting, the difficulties in preserving renal blood flow while accomplishing surgical repair can be appreciated.

Preoperative contrast urography (intravenous pyelography [IVP]) prior to all abdominal aortic aneurysm surgery to identify such anomalies has been advocated. With the increasing use of ultrasonography to detect and evaluate abdominal aortic aneurysms, routine aortography (which might delineate such an anomaly) is falling into disuse. At our institution, IVP and aortography are seldom used for routine abdominal aortic aneurysm surgery. The risk of renal injury secondary to the contrast dye load and the potential for complications from further invasive diagnostic procedures are 
factors contributing to this trend. Clot within or surrounding the aneurysm may preclude the diagnosis of horseshoe kidney by ultrasonography. ${ }^{16}$ Retrospective review of the aortic ultrasonogram in the case reported here failed to indicate horseshoe kidney, even in light of the operative findings.

When the anomaly is encountered unexpectedly at surgery, it poses more of a dilemma, since the arterial supply to the kidney is variable and undocumented. If the blood supply is not readily apparent at dissection, intraoperative aortography may be employed. ${ }^{27}$ If consideration is given to ligation of small anomalous vessels, or if anomalous vessels are inadvertently ligated or severed, adequate perfusion to the involved renal segment must be assured, or the segment must be resected. ${ }^{6,17}$ Anomalous arteries should be preserved whenever possible. If involved in the aneurysm, a "button" of aortic wall surrounding the origin of the vessel (or vessels) can be excised and reimplanted onto the aortic graft. ${ }^{17}$ Perfusion of the excised accessory vessels can be maintained during repair by the use of heparin-bonded shunts from the iliac arteries. ${ }^{6}$

Division of the isthmus (symphysiotomy) or resection (symphysiectomy) generally should be avoided, although circumstances may dictate otherwise. Symphysiectomy is preferred to symphysiotomy, although both introduce the risk of graft infection (from exposure to chronically infected urine) and urinary fistula. ${ }^{17}$

Starr and coworkers ${ }^{19}$ listed three factors that influence the difficulty of aneurysmectomy with horseshoe kidney: (1) the consistency of the renal isthmus; (2) the pattern of blood supply; and (3) the origin of the arteries supplying the isthmus. In the ideal technical situation, the isthmus is thin and fibrous and there are no anomalous renal arteries. These conditions, unfortunately, are not often met. As a result, the complexity of aortic reconstruction taxes the ingenuity of even the experienced surgeon.

\section{Summary}

Horseshoe kidney coexisting with abdominal aortic aneurysm is an unusual surgical problem. Whether diagnosed preoperatively or encountered at laparotomy, it requires thoughtful operative management. The goals of the surgeon must include adequate aneurysm repair and preservation of the often anomalous blood supply to the fused renal segment.

1. Bietz, D.S., and Merendino, K.A.: Abdominal aneurysm and horseshoe kidney. A review. Ann Surg 181:333-41, Mar 75

2. Brown, O.W., Dosick, S.M., and Blakemore, W.S.: Abdominal aortic aneurysm and horseshoe kidney. A different perspective. Arch Surg
114:860-1, Jul 79

3. Brown, L., Martinez, L., and Mannix, H., Jr.: Abdominal aortic aneurysm and horseshoe kidney in a 91-year-old man. Am Surg 43:531-3, Aug 77

4. Bugge-Asperheim, B., Birkeland, S., and Helgerud, P.: Horseshoe kidney and ruptured abdominal aortic aneurysm. Scand J Urol Nephrol 11:89-92, 1977

5. Connelly, T.L., et al.: Abdominal aortic surgery and horseshoe kidney Report of six cases and a review. Arch Surg 115:1459-63, Dec 80

6. Cox, J.L., and Sabiston, D.C., Jr.: The use of heparin bonded shunts for perfusion of the renal artery during resection of complex abdominal aortic aneurysms. Surg Gynecol Obstet 147:859-64, Dec 78

7. Donati, A., et al.: Abdominal aortic aneurysm and horseshoe kidney. J Cardiovasc Surg 21:632-6, Sep-Oct 80

8. Du Toit, D.F., et al.: Ruptured abdominal aortic aneurysm and horseshoe kidney. A case report. S Afr Med J 64:750-1, Oct 83

9. Ezzet, F., Dorazio, R., and Herzberg, R.: Horseshoe and pelvic kidneys associated with abdominal aortic aneurysms. Am J Surg 134:196-8, Aug 77

10. Van Gelderen, P.W., and Brands, L.C.: Ruptured aneurysm of the abdominal aorta in the presence of a horseshoe kidney. Arch Chir Neerl 27:271-6, 1975

11. Gillet, M., et al: Anévrysme de l'aorte abdominale rompu dans le veine cave infériure et rein en fer à cheval. Chirurgie 108:811-5, 1982

12. Gutowicz. M.A., and Smullens, S.N.: Ruptured abdominal aortic aneurysm with horseshoe kidney. J Vasc Surg 1:689-91, Sep 84

13. Landes, R.G., Trumbull, H.R., and Nicoloff, D.M.: Abdominal aortic aneurysm with rupture into the inferior vena cava associated with horseshoe kidney. Ann Surg 187:329-31, Mar 78

14. Louagie, Y.A., et al.: Horseshoe kidney and abdominal aortic aneurysm. Acta Chir Belg 84:249-54, Jul-Aug 84

15. Poulias, G.E., et al.: Successful resection of ruptured abdominal aortic aneurysm into the inferior vena cava, in the presence of a horseshoe kidney. Case report and review of the literature. J Cardiovasc Surg 23:415-21, Sep-Oct 82

16. Rosenfield, A.T., Lowman, R.M., and Taylor, KJW: Urography in preoprative evaluation of abdominal aortic aneurysms. Urology 7:652-4, Jun 76

17. Sidell, P.M., et al.: Horseshoe kidney associated with surgery of the abdominal aorta. Mayo Clin Proc 54:97-103, Feb 79

18. Soriano, J., et al: Abdominal aortic aneurysm and the horseshoe kidney. J Med Soc NJ 73:584-8, Jul 76

19. Starr, D.S., Foster, W.J., and Morris, G.C., Jr.: Resection of abdominal aortic aneurysm in the presence of horseshoe kidney. Surgery 89:387-9, Mar 81

20. Pender, D.J.: Ruptured abdominal aortic aneurysm with horseshoe kidney. Brit J Hosp Med 35:414-5, Jun 86

21. Constantino, M.J.: Horseshoe kidney associated with aortic aneurysm. J Med Assoc Georgia 75:212-4, Apr 86

22. Hardin, C.A.: Abdominal aortic aneurysm and horseshoe kidney. Kansas Med 87:274-5, Oct 86

23. Perlmutter, A.D., Retik, A.B., and Bauer, S.B.: Anomalies of the upper urinary tract. In Campbell's Urology, edited by P.C. Walsh, et al. Ed. 5. W.B. Saunders Co., Philadelphia, 1986

24. Sadler, T.W.: Langman's Medical embryology. Ed. 5. Williams \& Wilkins Co., Baltimore, 1985

25. Boatman, D.L., Cornell, S.H., and Kolln, C-P.: The arterial supply of horseshoe kidneys. Am J Roentgenol Rad Ther Nuc Med 113:447-51, Nov 71

26. Graves, F.T.: The arterial anatomy of the congenital abnormal kidney. Br J Surg 56:533-41, Jul 69

27. Rutherford, R.B.: Infrarenal aortic aneurysms. In Vascular surgery, edited by R.B. Rutherford. Ed. 2. W.B. Saunders Co., Philadelphia, 1984

At the time this paper was written, Dr. Waters was chief resident in general surgery, Des Moines General Hospital. The residency program director is Norman Rose, D.O., FACOS. He is now a fellow in cardiovascular surgery, Cleveland Clinic Foundation, Cleveland, Ohio. Dr. Esper was a resident in general vascular surgery at DMGH, where Dr. Stanley is the residency program director and a staff cardiovascular surgeon.

Dr. Waters, Cleveland Clinic Educational Foundation, Box 596 , 9500 Euclid Avenue, Cleveland, Ohio 44106. 


\section{American Osteopathic Association \\ Continuing Medical Education}

\section{CERTIFICATION OF HOME STUDY}

This is to certify that I, activity for AOA CME credits.

Please print

completed the following

Type of activity (such as reading or listening)

Name of journal(s) or audio-tape and date(s) of issue(s):

(One-half CREDIT may be granted for each issue or tape)

AOA number

College and year of graduation
D.O.'s signature

MAIL TO: AOA Division of CME, 212 East Ohio Street, Chicago, Illinois 60611

KEEP A DUPLICATE FOR YOUR RECORDS!

The Home Study form is intended to document individual reading of recognized scientific journals, listening to approved audio-tapes, and other approved home study courses and programs under the criteria described for Category 2-B.

Only one type of home study, such as reading, should be indicated on a single form, though multiple issues of scientific journals may be listed.

This form should not be used, however, when CME quiz cards for the AOA Journal are submitted separately.

\begin{tabular}{|l|}
\hline FOR OFFICE USE ONLY \\
\hline Cat. $\longrightarrow$ Credits \\
Program $\#$ 2-B \\
Doctor \# \\
Doctor's Name \\
\hline
\end{tabular}

Please refer to the revised CME GUIDE for additional information. 


\section{non-insulin-dependent}

\section{Diabetes and fasting glucose}

Fasting hyperglycemia is largely due to hepatic overproduction of glucose. MiCRONASE has been shown to decrease elevated basal rates of hepatic glucose production, and this may account for its ability to reduce fasting hyperglycemia.

MICRONASE improves 24-hour control of both postprandial and fasting blood glucose levels. MicRONASE usually provides 24-hour control of blood glucose levels with a once-a-day dosage.

All sulfonylureas, including MiCRONASE, can cause severe hypoglycemia. Proper patient selection, dosage, and instructions are important.

No other oral antidiabetic agent fits the realities of life better than

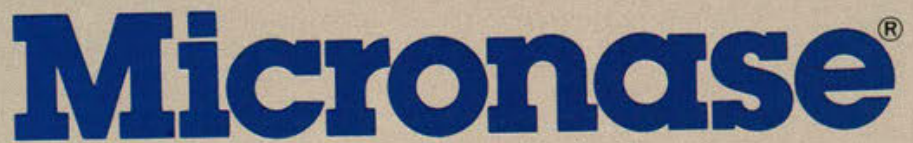

\section{Tablets (glyburide)}

Usual starting dosage $-2.5 \mathrm{mg}$ to $5 \mathrm{mg}$ once a day

When diet alone fails...MICRONASE

Please see adjacent page for brief summary of prescribing information.

\section{Upjohn}



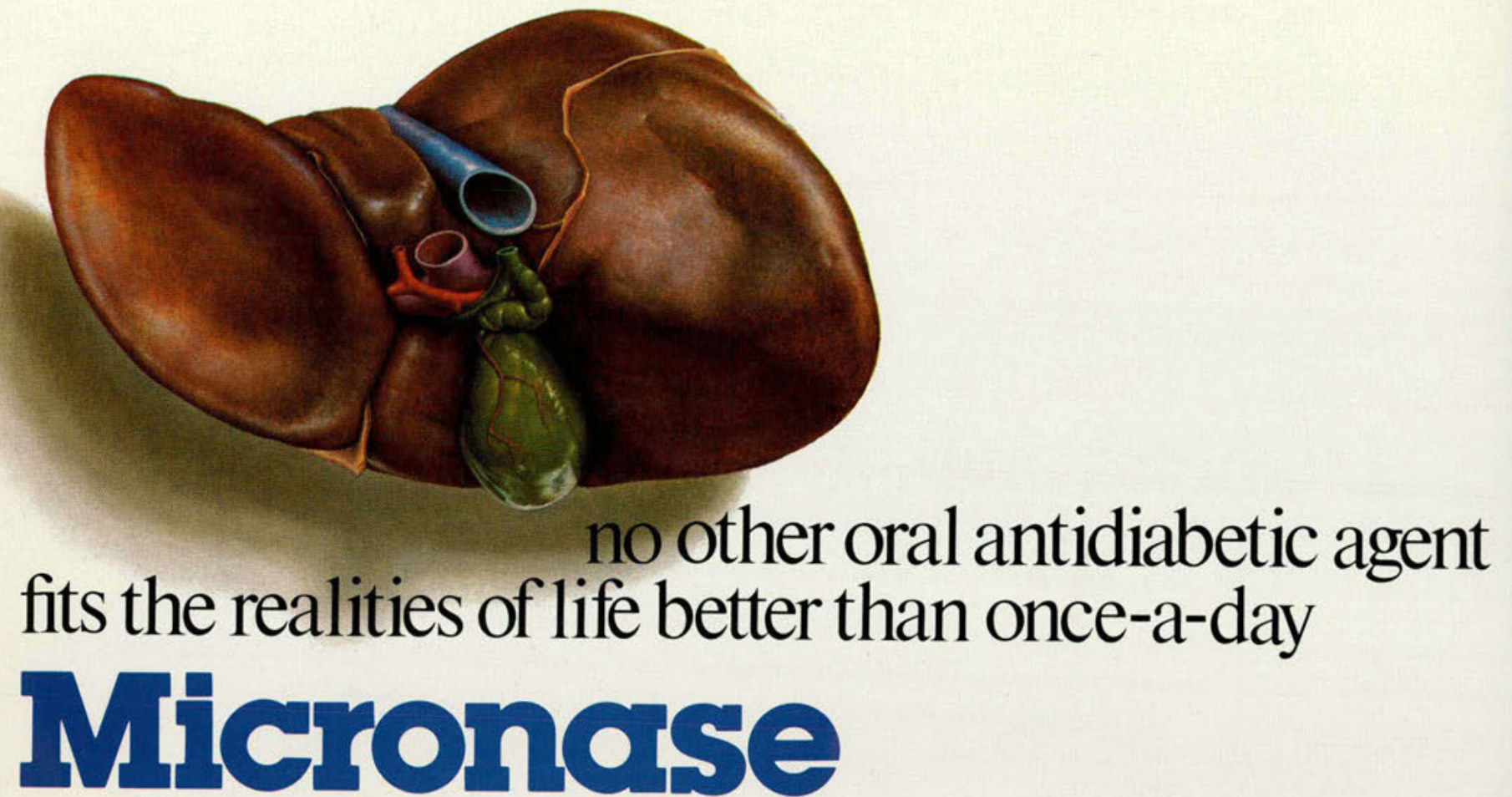

Some patients, particularly those receiving more than $10 \mathrm{mg}$ daily, may have a more satisfactory response with twice-a-day dosage.

\section{Micronase Tablets (glyburide)}

CONTRAINDICATIONS: MICRONASE Tablets are contraindicated in patients with: 1 . Known hypersensitivity or allergy to the drug. 2. Diabetic ketoacidosis, with or without coma. This condition should be treated with insulin. 3. Type I diabetes mellitus. as sole therapy.

SPECIAL WARNING ON INCREASED RISK OF CARDIOVASCULAR MORTALITY: The administration of oral hypoglycemic drugs has been reported to be associated with increased cardiovascular mortality as compared to treatment with diet alone or diet plus insulin. This warning is based on the study conducted by the University Group Diabetes Program (UGDP), a long-term prospective clinical trial designed to evaluate the effectiveness of glucose-lowering drugs in preventing or delaying vascular complications in patients with noninsulin-dependent diabetes. The study involved 823 patients who were randomly assigned to one with noninsulin-dependent diabetes. The study involved 823 pation
of four treatment groups (Diabetes, 19 [Suppl 2]:747-830, 1970.

UGDP reported that patients treated for 5 to 8 years with diet plus a fixed dose of tolbutamide (1.5 grams per day) had a rate of cardiovascular mortality approximately $2 \frac{1}{2}$ times that of patients treated with-diet a pere A significant increase in total mortality was not observed, but the use of tolbutamide was discontine. based on the increase in cardiovascular mortality, thus limiting the opportunity for the study to show an increase in overall mortality. Despite controversy regarding the interpetation of these results io show an of the UGDP study provide an adequate basis for this warning. The patient should be informed of the potential risks and advantages of MICRONASE and of alternative modes of therapy.

Although only one drug in the sulfonylurea class (tolbutamide) was included in this study, it is prudent from a safety standpoint to consider that this warning may apply to other oral hypoglycemic drugs in this class, in view of their close similarities in mode of action and chemical structure.

PRECAUTIONS: General-Hypoglycemia: All sulfonylureas are capable of producing severe hypoglycemia Proper patient selection and dosage and instructions are important to avoid hypoglycemic episodes. Renal or hepatic insufficiency may increase the risk of serious hypoglycemic reactions. Elderly, debilitated of malnourished patients, and those with adrenal or pituitary insufficiency, are particularly susceptible to the hypoglycemic action of glucose-lowering drugs. Hypoglycemia may be difficult to recognize in the elderly and in people who are taking beta-adrenergic blocking drugs. Hypoglycemia is more likely to occur when caloric intake is deficient, after severe or prolonged exercise, when alcohol is ingested, or when more than one glucose lowering drug is used. Loss of Control of Blood Glucose- In diabetic patients exposed to stress such as fever, trauma infection or surgery a loss of control may occur It may then be nocessary discontinue MICRONASE and administer insulin Adeouate adiustment of dose and adher be nocossary to

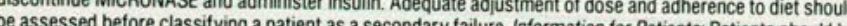
informed of the potential risks and advant as a secondary lailure. Information for Patients. Patients should be informed of the potential risks and advantages of MICRONASE and of alternative modes of therapy. They also should be informed about the importance of adherence to dietary instructions, of a regular exercise program. and of regular testing of urine and/or blood glucose. The risks of hypoglycemia, its symptoms and treatment and conditions that predispose to its development should be explained to patients and responsible family members. Primary and secondary failure should also be explained laboratory Tests: Response MICRONASE Tablets should be monitored by trequent urine olucose tests and periodic blood alusponse to Measurement of glycosylated hemoglobin levels may be helpful in some patients. Drug Interactions: The hypoglycemic action of sulfonylureas may be potentiated by certain drugs including nonsteroidal antiinflammatory agents and other drugs that are highly protein bound, salicylates, sulfonamides, chloramphencol, probenecid, coumarins, monoamine oxidase inhibitors, and beta adrenergic blocking agents. Certain drugs tend to produce hyperglycemia and may lead to loss of control. These drugs include the thiazides and other diuretics, corticosteroids, phenothiazines, thyroid products, estrogens, oral contraceptives, phenytoin, nicotinic acid, sympathomimetics, calcium channel blocking drugs, and isoniazid. A potential interaction between oral miconazole and oral hypoglycemic agents leading to severe hypoglycemis has been reported Carcinogenesis, Mutagenesis, and lmpairment of fert lity: Studies in rats at doses up $10300 \mathrm{mg} / \mathrm{kg} / \mathrm{by}$ lor 18 months showed no carcinogenic effects. Glyburide is nonmutagenic when studied in the Salmonella microsome test (Ames test) and in the DNA damage/alkaline elution assay. Pregnancy: Teratogenic Effects. Pregnancy Category B. Reproduction studies in rats and rabbits have revealed no evidence of impaired fertility or harm to the fetus due to glyburide. There are no adequate and well controlled studies in pregnan women. This drug should be used during pregnancy only if clearly needed. Insulin should be used during pregnancy to maintain blood glucose as close to normal as possible. Nonteratogenic Effects: Prolonged severe hypoglycemia ( 4 to 10 days) has been reported in neonates born to mothers who were receiving a sulfonylurea drug at the time of delivery. MICRONASE should be discontinued at least two weeks before the expected delivery date Nursing Mothers: Some sulfonylurea drugs are known to be excreted in human milk. Insulin therapy should be considered. Pediatric Use: Safety and effectiveness in children have not been established.

ADVERSE REACTIONS: Hypoglycemia: See Precautions and Overdosage sections. Gastrointestinal Reactions: Cholestatic jaundice may occur rarely: MICRONASE Tablets should be discontinued if this occurs. Gastrointestinal disturbances, e.g. nausea, epigastric fullness, and heartburn, are the most common reactions, having occurred in $1.8 \%$ of treated patients during clinical trials. They tend to be dose-related and may disappear when dosage is reduced. Liver function abnormalities, including isolated transaminase elevations, have been reported Dermator rticaria and morbiliform or macul rials. These mar be tran or acul mapular eruptions, occurred in $1.5 \%$ of treated patients during clinical trials. These may be transient and may disappear despite continued use of MICRONASE; if skin reactions persist, the drug should be discontinued. Porphyria cutanea tarda and photosensitivity reactions have been reported with sulfonylureas. Hematologic Reactions: Leukopenia, agranulocytosis, thrombocytopenia, hemolytic anemia, aplastic anemia, and pancytopenia have been reported with sulfonylureas. Metabolic Reactions: Hepatic porphyria and disulfiram-like reactions have been reported with sulfonylureas; however hepatic porphyria has not been reported with MICRONASE and disulfiram-like reactions have been reported very rarely. Cases of hyponatremia have been reported with glyburide and all other sulfonylureas. most then in patients who are on other medications or crave medical conditions known al o cas sullonyloreas, most often release of antidiuretic hormone (SIADH) secretion has been rioported with certain ther suttria or increase it has been suggested that these sulfonylureas may augment the peripheral (antidiuretic) action of $\mathrm{ADH}$ and lor increase release of $A D H$.

OVERDOSAGE: Overdosage of sulfonylureas, including MICRONASE Tablets, can produce hypoglycemia. If hypoglycemic coma is diagnosed or suspected, the patient should be given a rapid intravenous injection of concentrated (50\%) glucose solution. This should be followed by a continuous infusion of a more dilute $(10 \%)$ glucose solution at a rate which will maintain the blood glucose at a level above $100 \mathrm{mg} / \mathrm{dL}$. Patients should be closely monitored for a minimum of 24 to 48 hours, since hypoglycemia may recur after apparent clinical recovery.

Caution Federal law prohibits dispensing without prescription. Store at controlled room temperature $15^{\circ}-30^{\circ} \mathrm{C}\left(59^{\circ}-86^{\circ} \mathrm{F}\right)$. Dispensed in well closed containers with safety closures. Keep container tightly closed For additional product information see your Upjohn representative.

\section{Upjohn}

THE UPJOHN COMPANY, Kalamazo0, MI 49001, USA 